\title{
Micropapillary Structures in Colorectal Cancer: An Anoikis-resistant Subpopulation
}

\author{
MADHURA PATANKAR $^{1,2}$, SARA VÄYRYNEN ${ }^{1,2}$, ANNE TUOMISTO $^{1,2,3}$, \\ MARKUS MÄKINEN ${ }^{1,2,3}$, SINIKKA ESKELINEN ${ }^{1}$ and TUOMO J. KARTTUNEN ${ }^{1,2,3}$ \\ ${ }^{1}$ Cancer and Translational Medicine Research Unit, University of Oulu, Oulu, Finland; \\ ${ }^{2}$ Medical Research Center Oulu, University of Oulu, Oulu, Finland; \\ ${ }^{3}$ Oulu University Hospital, Department of Pathology, Oulu, Finland
}

\begin{abstract}
Background/Aim: Micropapillary structures (MIPs) are focal piles of columnar cells without extracellular matrix contact, and common in serrated colorectal carcinoma (CRC). In order to characterize biology of MIPs in colorectal cancer $(C R C)$, the proliferation and apoptosis rates, and survivin expression were compared between MIPs and other cancer epithelial cells of CRC (non-MIPs). Materials and Methods: We assessed 46 samples of normal colorectal mucosa, 62 carcinomas and 54 polyps for proliferation (Ki67), apoptosis (M30), and survivin expression by immunohistochemistry. Results: MIPs in carcinoma showed lower rates of proliferation and apoptosis than non-MIPs. A low rate of apotosis in MIPs was associated with poor prognosis in local carcinoma. In normal crypts, nuclear-to-cytoplasmic transition of survivin indicated epithelial cell maturation. Cancer cases showed increased cytoplasmic expression of survivin than normal mucosa and polyps. However, MIPs showed lower nuclear and cytoplasmic survivin expression than non-MIPs. Our findings suggest that MIPs represent a biologically distinct subpopulation of carcinoma cells with features of anoikis resistance and possibly quiescence.
\end{abstract}

About $10-30 \%$ of colorectal carcinomas $(\mathrm{CRC})(1,2)$ are serrated adenocarcinomas with predominance of $K R A S$ or $B R A F$ mutations and poor prognosis (3-5).The presence of focal epithelial cell piles without fibrovascular core is a characteristic feature of serrated CRC type (1, 4, 6-9). These structures are analogous to micropapillary structures (MIPs). There is no generally accepted definition of MIPs but they

This article is freely accessible online.

Correspondence to: Professor Tuomo J. Karttunen, PO Box 5000, 90014, Oulu Finland. E-mail: tuomo.karttunen@oulu.fi

Key Words: Serrated adenocarcinoma, colon, micropapillary structure, survivin, proliferation, apoptosis, anoikis resistance. are usually defined as groups of epithelial cells piled up on each other and usually lacking a fibrovascular core. MIPs generally form luminal extensions in the glands (Figure 1). It should be noted that the so-called micropapillary carcinomas are composed of epithelial cell clusters floating freely in clear spaces (10). These structures clearly differ from the current classical concept of MIPs, where the cell piles are directed to the luminal side and are connected to columnar tumor epithelium. In addition to CRC, MIPs as defined above are present in other carcinoma types, including ovarian and lung carcinomas, where they are similarly associated with worse prognosis $(11,12)$. However, the biology of MIPs has not been characterized. We noted that within the MIPs in CRC there are no extracellular basement membrane proteins (unpublished data), suggesting that the suprabasal cells of the MIPs do not have contact with the extracellular matrix.

Survivin is a member of the inhibitor of apoptosis protein family which regulates cell proliferation and apoptosis (1315). Survivin is up-regulated in most cancer types (16-18) and its high expression is associated with adverse prognosis (19). Nuclear and cytoplasmic expression of survivin have different prognostic significance $(18,20,21)$. Survivin expression in different subpopulations of cells has not been studied in any carcinoma typeto our knowledge.

We hypothesized that the suprabasal cells in the MIPs might form a functionally distinct subpopulation of tumor epithelial cells in CRC, characterized by the adaptation of being able to survive without contact with the extracellular matrix, and accordingly, might be resistant to anoikis, i.e. apoptosis induced by loss of extracellular matrix contact $(22,23)$. Based on the importance of anoikis resistance in metastasis formation (22), the presence of such a subpopulation could have prognostic significance. In order to characterize possible anoikis resistance in this cell subpopulation, we compared indices for proliferation (PI) and apoptosis (AI) in MIP and non-MIP epithelium of colorectal tumors and studied expression of survivin in relation to anoikis inhibition. 
Table I. Patient characteristics.

\begin{tabular}{lccc}
\hline Lesion type & $\begin{array}{r}\text { Number } \\
\text { of cases }\end{array}$ & $\begin{array}{c}\text { Mean age } \\
\text { (range), years }\end{array}$ & $\begin{array}{c}\text { Male/female, } \\
\mathrm{n}\end{array}$ \\
\hline Normal mucosa & 59 & $65(47-84)$ & $31 / 28$ \\
Polyps (All) & 56 & & \\
Serrated polyps & 35 & $63(37-86)$ & $12 / 23$ \\
Serrated adenomas & 25 & $66(45-81)$ & $9 / 16$ \\
Hyperplastic polyps & 10 & $62(48-85)$ & $3 / 7$ \\
Conventional adenomas & 21 & $65(45-81)$ & $13 / 8$ \\
Carcinomas & 59 & & \\
Serrated & 28 & $65(45-81)$ & $13 / 15$ \\
Conventional & 31 & $63(48-85)$ & $18 / 13$ \\
\hline
\end{tabular}

\section{Materials and Methods}

Sample collection. A case series was collected from the Department of Pathology, Oulu University Hospital, and included 59 samples of normal mucosa, 56 benign polyps and 59 carcinomas (Table I). We aimed to obtain a representative series of serrated carcinomas $(n=28)$ and comparable number of unselected conventional carcinomas $(n=31)$ matched by gender, location, Dukes' stage and WHO histological grade $(5,6)$. The research project was approved by the Ethical Council of Oulu University Hospital (25/2002, $58 / 2005,184 / 2009)$.

Immunohistochemistry. For immunohistochemistry (24), sections were made from formalin-fixed paraffin-embedded specimens and antigen retrieval was performed with Tris-EDTA buffer, $\mathrm{pH} 9$ in a microwave ( $800 \mathrm{~W}$ for $2 \mathrm{~min}, 150 \mathrm{~W}$ for $15 \mathrm{mins}$ ). Antibodies for Ki67 (code NCL-Ki67-MM1, dilution 1/50; Leica Biosystems, Newcastle-upon-Tyne, UK) and M30 (CytoDEATH cat.no. 12140322001, dilution 1/1,000; Enzo Life Sciences, Lausen, Switzerland) were incubated for $30 \mathrm{~min}$ at room temperature. For survivin staining, the antigen retrieval used citrate buffer at $\mathrm{pH} 6$ ( $800 \mathrm{~W}$ for $2 \mathrm{~min}, 150 \mathrm{~W}$ for $15 \mathrm{~min}$ ) followed by rabbit polyclonal anti-survivin (Abcam469, dilution 1/1000; Abcam, Cambridge, UK) incubated for $30 \mathrm{~min}$ at room temperature. Envision (K5007; Dako, Copenhagen, Denmark) was used for detection of bound antibodies. For controls, PBS instead of primary antibodies was used.

Definition of MIPs and assessment of immunohistochemical staining. Two independent observers (MP and SV) blinded for clinical and pathological information evaluated the sections. MIPs were defined as luminal extensions formed by a group of epithelial cells alone piled at least two cells thick (Figure 1), without any fibrovascular core. In such a pile, only the basal layer is in contact with the extracellular matrix.

Staining patterns were separately assessed in the suprabasal cells of the MIPs if present and in the non-MIP epithelium. PI and AI were determined according to the proportion of cells with nuclear expression of $\mathrm{Ki} 67$ and cytoplasmic expression of M30, respectively. Survivin expression was separately assessed in the cytoplasm and nucleus, and both the percentage of positively stained cells and the intensity of staining (negative: 0 ; weak: 1 ; moderate: 2; strong: 3) were recorded. However, as there was no variation in

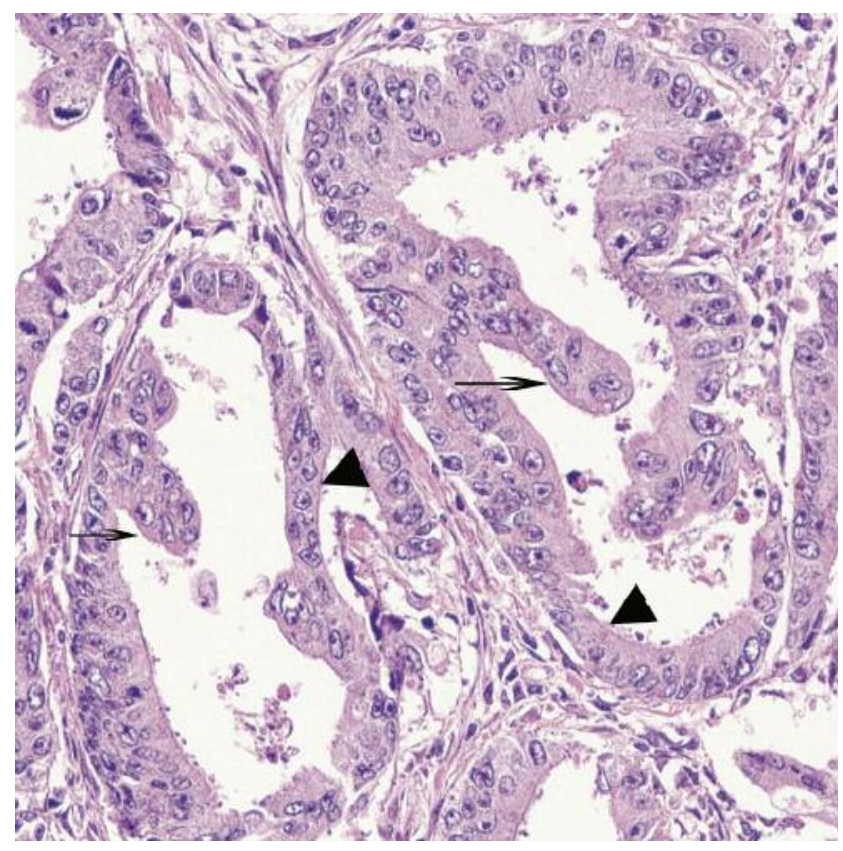

Figure 1. Microphotograph showing colorectal carcinoma with micropapillary structures (MIPs). MIPs are formed from piles of epithelial cells extending into the lumen (arrows). Due to tangential sectioning, patches where epithelium seems to form multilayered foci can occasionally be seen (arrowheads). However, such foci can usually be differentiated from true MIPs by observing the lack of clear lateral bordering of such areas. Original magnification, $\times 400$.

the intensity of staining, only the proportion of positively stained cells was used for this study. For all assessments, the average of the scores of the observers was used. The cases with significant disagreement ( $\geq 30 \%$ in proportion; $>1$ point in intensity) were reevaluated and consensus was reached. In normal mucosa and polyps, the upper and lower half of mucosa, and in carcinomas, the invasive front and tumor bulk were separately evaluated. The results are reported as median (range) values.

Statistical analysis. IBM SPSS 22 (IBM Corp., Armonk, NY, USA) software was used for statistical analyses. Mann-Whitney and Kruskal-Wallis tests were used to compare lesion types and the Wilcoxon matched-pair test for comparing different parts of the lesions. $p$-Values of less than 0.05 were considered significant. Spearman rank correlation test was used for studying correlations of PI, AI and survivin expression. Associations with clinicopathological features were analyzed with the Mann-Whitney, Kaplan-Meier, and log-rank tests.

\section{Results}

PI, AI and survivin expression in normal colorectal mucosa. As expected, epithelial cells in the upper half of normal mucosa had a lower PI [median (range): upper part: 1 (0-23); lower: 23 (4-45); $p<0.001]$ and higher AI [upper part: 2 (0- 

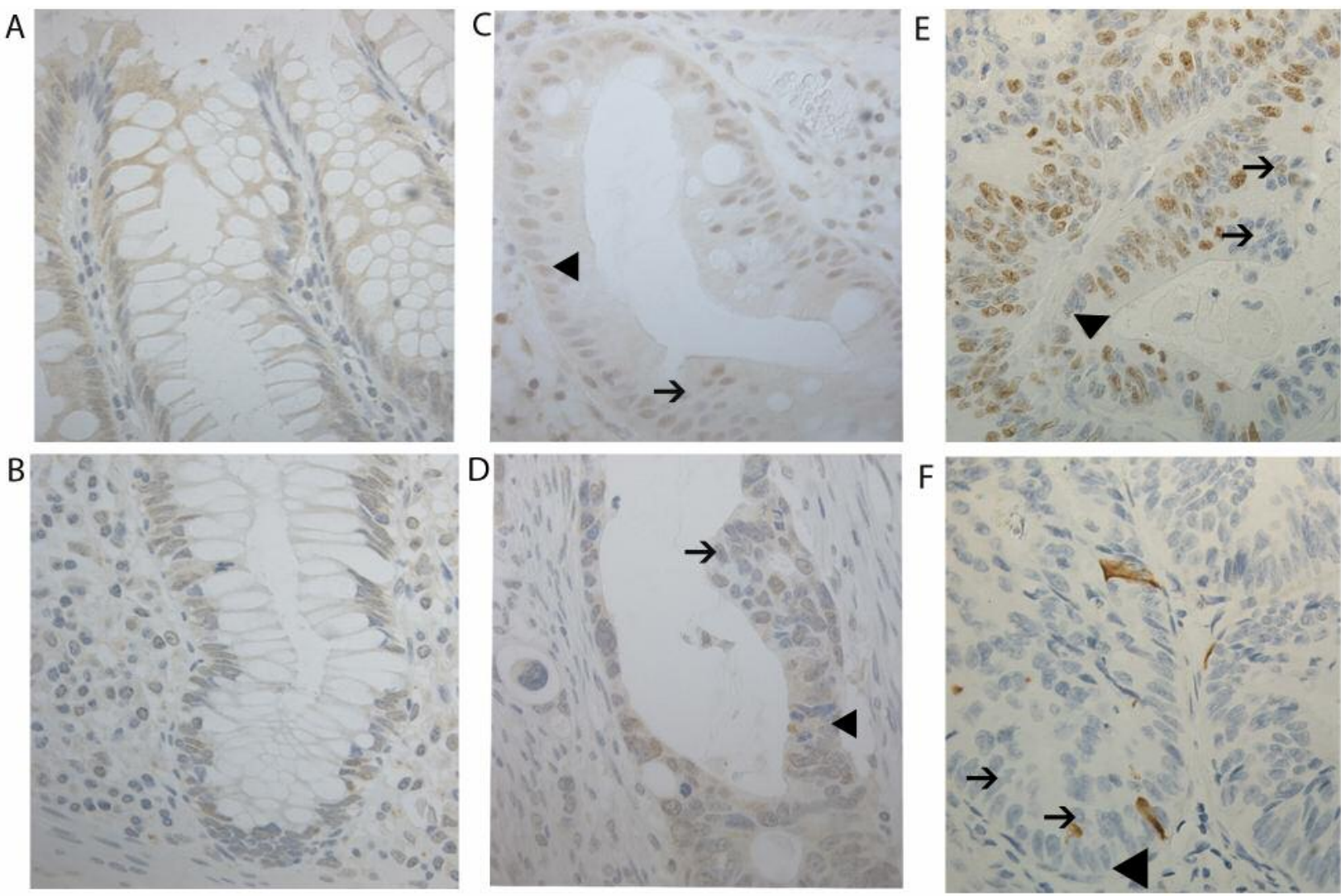

Figure 2. Microphotographs showing expression of survivin (A-D), Ki67 (E), and M30 (F) in colon. Normal mucosa exhibited predominantly cytoplasmic survivin expression in the upper half $(A)$ and predominantly nuclear expression in the lower half $(B)$ of the crypt. Micropapillary structures (MIPs; arrows) exhibit less survivin expression as compared with the non-MIP epithelium (arrowheads) in both serrated polyp (C) and serrated colorectal carcinoma (D). In colorectal serrated carcinoma, MIPs (arrows) have fewer cells with Ki67 expression (E) or M30 expression (F) than the non-MIP cells. Original magnification, $\times 400$.

50); lower part: $1(0-38) ; p<0.001]$. Nuclear survivin staining was higher (proportion of positively stained cells) in the lower half [median (range), 65\% (2-93\%) vs. 45\% (595\%), respectively; $p<0.001]$, whereas cytoplasmic expression was higher in the upper half $[58 \%(0-100 \%) v s$. $0 \%(0-100 \%) ; p<0.001]$; (Figure 2).

PI and AI in MIP and non-MIP epithelium. MIPs (Figure 1) were mostly seen in the serrated lesions, but were occasionally found in conventional ones. In both polyps and carcinomas, PI and AI were lower in the MIP than in the non-MIP epithelium (Table II; Figure 2), and the difference was similarly evident in both conventional and serrated polyps, and carcinomas. Interestingly, the AI/PI ratio was lower in the MIP cells than in the non-MIP cells (Table II). $\mathrm{PI}, \mathrm{AI}$ and the AI/PI ratio in the MIPs correlated with those in the non-MIP cells both in polyps and carcinomas (polyps: PI, $p=0.02$; AI, $p<0.001$; AI/PI, $p<0.001$; carcinoma: PI, $p<0.001, \mathrm{AI}, p<0.001, \mathrm{AI} / \mathrm{PI}, p<0.001)$.
Conventional adenomas had higher overall PI in both MIP and non-MIP cells when compared to serrated polyps (Table II). There was no difference between serrated adenomas and hyperplastic polyps (data not shown). Carcinomas had lower $\mathrm{AI}$ and $\mathrm{AI} / \mathrm{PI}$ ratio than polyps only for the non-MIP cells (Table II).

Association of clinicopathological features with AI and PI in MIP and non-MIP epithelium in carcinomas. Carcinoma grade was not associated with PI or AI in MIP or non-MIP cells. Carcinoma stage also showed no association with PI or AI in the MIPs, but advanced stage was associated with low AI in non-MIP cells [median (range): stages A-B: 3.5 (040); stages C-D: $1.75(1-26.5) ; p=0.050]$. In serrated carcinomas, the AI/PI ratio for MIPs tended to be higher in stage C-D carcinomas than those of stage A-B [median (range): $0.01(0-0.11) v s .0(0-0.31)$, respectively; $p=0.053$ ].

Neither PI, AI nor the AI/PI ratio in MIP or non-MIP cells correlated with cancer-related survival among patients, nor 
Table II. Indices for proliferation (PI) and apoptosis (AI) and apoptosis to proliferation ratios (AI/PI) in micropapillary structure (MIP) and in non-MIP epithelium in colorectal lesions. Statistically significant differences are marked with letters. Results are presented as median (range).

\begin{tabular}{|c|c|c|c|c|c|c|c|c|c|}
\hline \multirow[b]{2}{*}{ Lesion type } & \multicolumn{3}{|c|}{ Non-MIP } & \multicolumn{3}{|c|}{ MIP } & \multicolumn{3}{|c|}{$p$-Value: MIP $v s$. non-MIP } \\
\hline & AI & PI & $\mathrm{AI} / \mathrm{PI}$ & AI & PI & $\mathrm{AI} / \mathrm{PI}$ & AI & PI & $\mathrm{AI} / \mathrm{PI}$ \\
\hline \multirow{9}{*}{$\begin{array}{l}\text { Polyps (all) } \\
\text { Serrated polyps } \\
\text { Conventional ad } \\
\text { Carcinomas (all) } \\
\text { Serrated } \\
\text { Conventional } \\
p \text {-Value }\end{array}$} & $3(0-24)$ & $30(7-52)$ & $0.1(0-0.3)$ & $1(0-4)$ & $28.5(0-75)$ & $0.02(0-0.25)$ & $<0.001$ & 0.02 & $<0.001$ \\
\hline & $2(0-10)$ & $28(7-50)$ & $0.1(0-0.3)$ & $0(0-4)$ & $25(0-75)$ & $0(0-0.25)$ & $<0.001$ & 0.046 & $<0.001$ \\
\hline & $3(1-24)^{a}$ & $42(16-52)^{\mathrm{a}}$ & $0.08(0-0.25)^{\mathrm{a}}$ & $1(0-3)^{\mathrm{a}}$ & $38(10-75)^{\mathrm{a}}$ & $0.03(0-0.1)^{\mathrm{a}}$ & $<0.001$ & 0.267 & 0.02 \\
\hline & $1(0-40)^{\mathrm{b}}$ & $30(0-94)^{b}$ & $0.05(0-5.71)^{\mathrm{b}}$ & $0(0-6)^{b}$ & $22(0-93)^{b}$ & $0(0-0.35)^{b}$ & $<0.001$ & $<0.001$ & $<0.001$ \\
\hline & $1(0-10)$ & $30.5(1-86)$ & $0.03(0-0.29)$ & $0(0-2)$ & $19.50(0-73)$ & $0(0-0.25)$ & 0.001 & 0.001 & 0.009 \\
\hline & $1(0-40)^{\mathrm{c}}$ & $30(0-94)^{\mathrm{c}}$ & $0.06(0-5.71)^{\mathrm{c}}$ & $0(0-6)^{\mathrm{c}}$ & $22(1-93)^{\mathrm{c}}$ & $0(0-0.35)^{\mathrm{c}}$ & $<0.001$ & 0.004 & $<0.001$ \\
\hline & $0.190^{\mathrm{a}}$ & $0.002^{\mathrm{a}}$ & $0.840^{\mathrm{a}}$ & $0.067^{\mathrm{a}}$ & $0.04^{\mathrm{a}}$ & $0.350^{\mathrm{a}}$ & & & \\
\hline & $0.019^{\mathrm{b}}$ & $0.587^{\mathrm{b}}$ & $0.01^{\mathrm{b}}$ & $0.128^{\mathrm{b}}$ & $0.512 \mathrm{~b}$ & $0.128^{\mathrm{b}}$ & & & \\
\hline & $0.787^{\mathrm{c}}$ & $0.934^{\mathrm{c}}$ & $0.404^{\mathrm{c}}$ & $0.730^{\mathrm{c}}$ & $0.862 \mathrm{c}$ & $0.646^{\mathrm{c}}$ & & & \\
\hline
\end{tabular}

${ }^{a} v s$. Serrated polyps; ${ }^{b} v s$. all polyps; ${ }^{c} v s$. serrated carcinomas.

in the subgroups of serrated and conventional carcinomas. However, survival analysis performed within the group with local disease (stages A-B) showed low AI (lower than median) in MIP cells or non-MIP cells to be associated with poor survival (log-rank test: MIP: $p=0.028$; non-MIP: $p=0.039$; Figure $3 \mathrm{~A}$ and B). No such difference was present in those with advanced-stage (stages C-D) cancer.

Association of survivin with proliferation, apoptosis, and clinicopathological features in carcinomas. Survivin expression was present in all cases. Since the intensity of survivin staining showed no variation, only the proportion of positively stained cells was used for comparison. Overall, nuclear survivin expression was lower in polyps and cancer than in normal mucosa (Table III). In a subgroup analysis, conventional adenomas exhibited higher nuclear survivin expression than hyperplastic polyps in the upper half of the mucosa $(p=0.002$; data not shown). Such a difference was not present for cytoplasmic survivin expression, and there was no difference between serrated adenomas and hyperplastic polyps in this regard. Serrated and non-serrated carcinomas did not differ in survivin expression.

Cells in the MIPs exhibited lower nuclear and cytoplasmic survivin expression than the non-MIP cells in both polyps and carcinomas (Table IV). In polyps, the nuclear to cytoplasmic ratio of survivin expression was higher in MIPs than non-MIPs $(p=0.01)$, but such a difference was not seen in carcinomas (Table IV). MIPs in polyps exhibited lower nuclear and cytoplasmic survivin expression than these structures in cancer $(p<0.001$; Table IV). The nuclear to cytoplasmic ratio of survivin in MIPs was higher in polyps than in cancer $(p=0.008)$, but no such difference was present in non-MIP cells. There was no correlation between survivin expression and AI or PI for any lesion type (data not shown). High nuclear to cytoplasmic ratio for survivin in the non-
Table III. Overall nuclear and cytoplasmic survivin expression (proportion of positively stained cells) in normal mucosa, polyps and carcinomas. Results are presented as median (range).

\begin{tabular}{lcc}
\hline & \multicolumn{2}{c}{ Survivin $(\%)$} \\
\cline { 2 - 3 } Lesion type & $\begin{array}{c}\text { Nuclear } \\
\text { Median (range) }\end{array}$ & $\begin{array}{c}\text { Cytoplasmic } \\
\text { Median (range) }\end{array}$ \\
\hline Normal mucosa & $55(3-91)$ & $35(0-100)$ \\
Polyps (all) & $45.5(3-90)^{\mathrm{a}}$ & $76.5(2-100)^{\mathrm{a}}$ \\
Serrated & $45(3-90)$ & $69(2-100)$ \\
Conventional adenomas & $47(21-65)$ & $79(2-100)$ \\
Carcinomas (all) & $43(4-89)^{\mathrm{bc}}$ & $73(0-100)^{\mathrm{bc}}$ \\
Serrated & $43(4-89)$ & $78(0-100)$ \\
Conventional & $42.5(18-78)$ & $73(0-100)$ \\
$p$-Value & $0.723^{\mathrm{a}}$ & $0.306^{\mathrm{a}}$ \\
& $0.012^{\mathrm{b}}$ & $0.001^{\mathrm{b}}$ \\
& $0.007^{\mathrm{c}}$ & $0.005^{\mathrm{c}}$ \\
\hline
\end{tabular}

${ }^{a}$ vs. Normal mucosa; ${ }^{b}$ ss. all polyps; ${ }^{c} v s$. normal mucosa.

MIPs was associated with low survival in those with stage $\mathrm{C}$ or D cancer (log-rank $p=0.029$; data not shown).

\section{Discussion}

Although MIPs are found in several cancer types and associated with poor prognosis $(11,12)$, their biology has not been studied. Our study shows, for the first time, that in $\mathrm{CRC}$, proliferation and apoptosis rate were lower in MIPs as compared with other epithelial cells of the tumors. Accordingly, our findings indicate that the cells in MIPs form a subpopulation of tumor cells with inhibition of anoikis and features of a quiescent state. In addition, we present evidence that a low AI in both MIP and non-MIP 

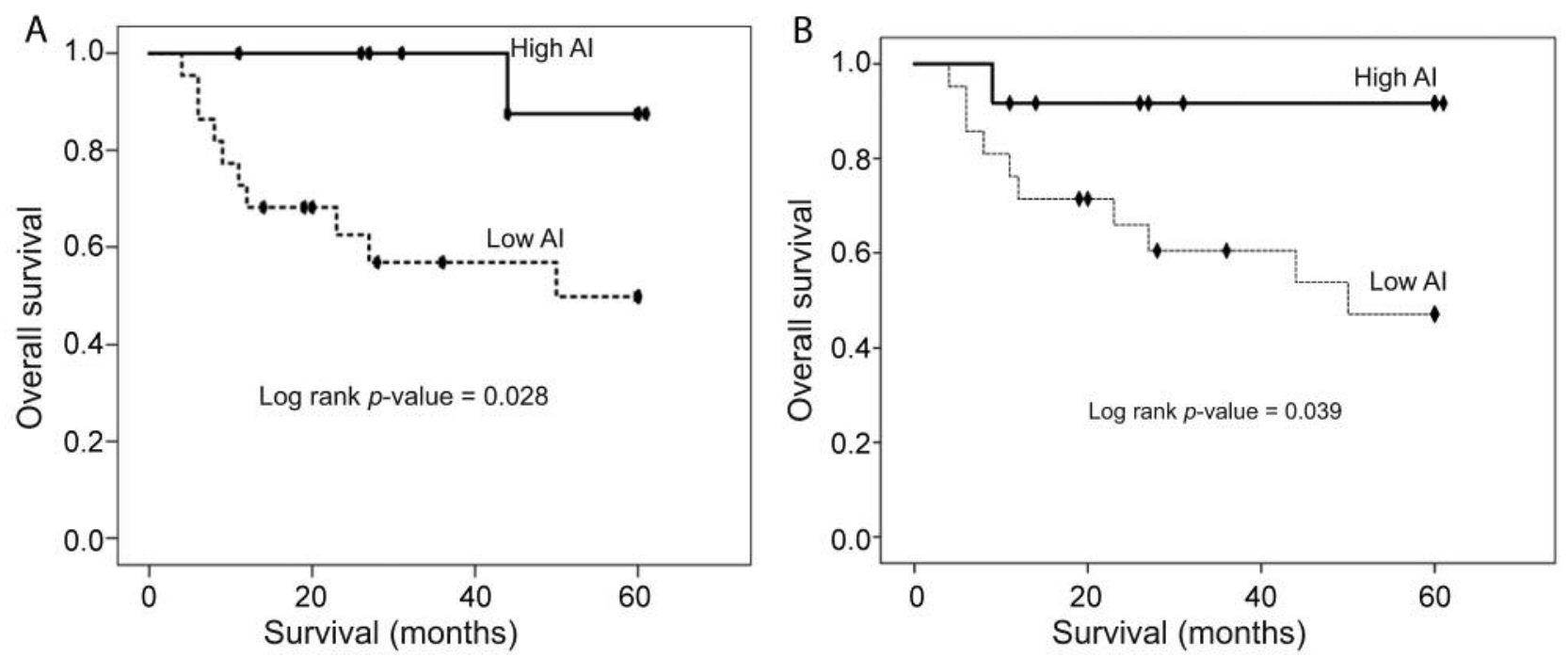

Figure 3. Kaplan-Meier curves for cancer-specific death according to low and high apoptosis index (AI) in the Micropapillary cells (MIPs) (A) and non-Micropapillary cells (non-MIPs) (B) among patients with early-stage colorectal cancer. Log-rank p-values are shown.

Table IV. Survivin expression (proportion of positive cells) compared in epithelium of micropapillary structures (MIPs) and in non-micropapillary regions (non-MIP) in colorectal polyps and carcinomas. Results are presented as median (range).

\begin{tabular}{|c|c|c|c|c|c|c|c|c|c|}
\hline \multirow[b]{3}{*}{ Lesion type } & \multicolumn{9}{|c|}{ Survivin $(\%)$} \\
\hline & \multicolumn{3}{|c|}{ Non-MIP } & \multicolumn{3}{|c|}{ MIP } & \multicolumn{3}{|c|}{$p$-Value: MIP $v s$. non-MIP } \\
\hline & Nucleus & Cytoplasm & $\mathrm{N} / \mathrm{C}$ ratio & Nucleus & Cytoplasm & $\mathrm{N} / \mathrm{C}$ ratio & Nucleus & Cytoplasm & $\mathrm{N} / \mathrm{C}$ ratio \\
\hline Polyps (all) & $45.5(3-90)$ & $65(2-100)$ & $0.56(0.06-14)$ & $1(1-3)$ & $1(0-3)$ & $1(0.5-3)$ & $<0.001$ & $<0.001$ & 0.001 \\
\hline Serrated polyps & $45(3-90)$ & $69(2-100)$ & $0.52(0.06-0.93)$ & $1(1-3)$ & $1(0-3)$ & $1(0.5-3)$ & $<0.001$ & $<0.001$ & 0.012 \\
\hline Conventional adenomas & $47(21-65)^{\mathrm{a}}$ & $79(2-100)^{a}$ & $0.57(0.39-14)^{\mathrm{a}}$ & $1(1-3)^{\mathrm{a}}$ & $2(1-2)^{\mathrm{a}}$ & $1(0.5-3)^{\mathrm{a}}$ & $<0.001$ & $<0.001$ & 0.035 \\
\hline Carcinomas (all) & $43(4-89)^{b}$ & $73(0-100)^{\mathrm{b}}$ & $0.51(0-15)^{\mathrm{b}}$ & $35(0-80)^{\mathrm{b}}$ & $62.5(0-100)^{b}$ & $0.53(0-3)^{b}$ & 0.026 & 0.012 & 0.757 \\
\hline Serrated & $43.5(4-89)$ & $75.5(0-100)$ & $0.41(0-15)$ & $30(0-80)$ & $67.5(0-100)$ & $0.27(0-1)$ & 0.04 & 0.235 & 0.206 \\
\hline Conventional & $42.5(18-78)^{\mathrm{c}}$ & $73(0-100)^{\mathrm{c}}$ & $0.55(0.31-12)^{\mathrm{c}}$ & $35(0-80)^{\mathrm{c}}$ & $52.5(0-100)^{\mathrm{c}}$ & $0.57(0.12-3)^{\mathrm{c}}$ & 0.259 & 0.240 & 0.465 \\
\hline \multirow[t]{3}{*}{$p$-Value } & $0.519^{\mathrm{a}}$ & $0.613^{\mathrm{a}}$ & $0.476^{\mathrm{a}}$ & $0.656^{\mathrm{a}}$ & $0.295^{\mathrm{a}}$ & $0.946^{\mathrm{a}}$ & & & \\
\hline & $0.723^{b}$ & $0.306^{b}$ & $0.753^{b}$ & $0.001^{b}$ & $0.001^{b}$ & $0.001^{b}$ & & & \\
\hline & $0.728^{c}$ & $0.855^{\mathrm{c}}$ & $0.412^{\mathrm{c}}$ & $0.383^{c}$ & $0.586^{\mathrm{c}}$ & $0.303^{c}$ & & & \\
\hline
\end{tabular}

$\mathrm{N} / \mathrm{C}$ ratio: Nuclear-cytoplasmic ratio. ${ }^{\mathrm{a}} v s$. Serrated polyps; ${ }^{\mathrm{b}}{ }_{v s .}$ all polyps; ${ }^{\mathrm{c}}$ vs. serrated carcinomas.

cells marks an adverse prognosis in local CRC. Finally, survivin expression was found reduced in MIPs, suggesting a back of this protein in the formation or maintenance of these structures.

MIPs are a characteristic of serrated carcinomas (6). Their pathogenesis in CRC, as well as in other malignancies, is unresolved, and both the mechanisms for initiation of cell pile formation and anoikis resistance in these structures reported here are unknown. Based on in vitro studies, general mechanisms for anoikis resistance include activation of prosurvival signaling, oncogene activation, growth factor receptor overexpression, and mutation/up-regulation of key factors involved in integrin or growth factor receptor signaling reviewed in (22). Since serrated carcinomas are associated with $K R A S$ or BRAF mutations (5), the mitogenactivated protein kinase mediated pro-survival activation associated with these mutations might be implicated in anoikis resistance in MIPs. Clearly, more studies are required to identify mechanisms which induce the formation and maintenance of MIPs.

Tumors with MIPs tend to confer a poor prognosis $(11,12)$ in an organ-independent fashion but the mechanisms of the 
prognostic effect have not been explored. Our study showing anoikis inhibition in MIPs suggests that this subset of tumor cells may be important for the formation of metastases, where anoikis inhibition is critical (23). Supporting this concept, we found that a low AI in MIPs was associated with adverse prognosis in localized CRC, however, along with similar association for a low AI in the non-MIP cells. An alternative explanation for cells in MIPs actually being metastatic is that the ability to form MIPs might be a marker of resistance to anoikis of the tumor epithelium. Low proliferation rates in MIPs is a feature of cell quiescence. As an alternative or additional factor explaining the prognostic effects of MIPs, quiescence may interfere with response to chemotherapy or radiotherapy (24-26). More studies are needed to assess whether the presence or characteristics of MIPs predicts oncological treatment responses.

Since survivin has a role in regulation of both apoptosis and proliferation $(15,16)$, we were interested in evaluating its possible role in the biology of MIPs. MIPs showed lower nuclear and cytoplasmic survivin expression than other tumor cells, supporting the idea that the cells in MIPs represent a functionally different subpopulation as compared with other cells of tumor epithelium. Interestingly, survivin expression was higher in MIPs of malignant lesions as compared to those of benign lesions, non-MIPs not showing such difference. Accordingly, an increase of survivin in MIPs seem to be associated with malignancy, and might be involved in the maintenance of these structures in carcinoma. However, survivin expression did not correlate with AI, PI or prognosis, leaving the biological significance of survivin in MIPs largely unconfirmed.

The overall role of survivin in colorectal mucosa and in colorectal cancer is controversial $(17,21)$. In our detailed analysis of normal mucosa, we found abundant nuclear survivin in the proliferating cells at crypt bases, a finding consistent with the role of nuclear survivin in cell proliferation $(15-17,27,28)$. Upper parts of normal mucosa showed increase of cytoplasmic survivin. Cytoplasmic survivin is generally associated with its anti-apoptotic role (18), and such an expression pattern in cells maturing for physiological apoptosis is unexpected. However, a complex network with pro-and anti-apoptotic signaling is present in the maturing crypt epithelium, and we speculate that nuclear to cytoplasmic transition of survivin forms a part of this network, contributing to an overall pro-apoptotic effect (29).

Serrated polyps had a bottom-heavy pattern of nuclear survivin expression, while conventional adenomas had surface-heavy pattern of survivin, as reported previously (30). Consistent with previous findings in CRC, carcinomas had higher cytoplasmic and lower nuclear survivin expression than normal colon mucosa or benign polyps (17, 19). As a new finding, serrated CRC showed no characteristic features for survivin expression.
In conclusion, we showed that AI in MIPs is lower than elsewhere in tumor epithelium. This finding indicates that the cells in the MIPs form a functionally separate subpopulation of tumor cells, which survives without contact with the extracellular matrix, i.e. shows inhibition of anoikis. A low PI in MIPs is a feature of quiescent stage. The importance of anoikis inhibition in metastasis (23) may explain our observation of the association between a low AI in tumor cell subpopulations both with and without contact with extracellular matrix and an adverse prognosis.

\section{Conflicts of Interest}

The Authors have declared no conflict of interest in regard to this study.

\section{Acknowledgements}

The Authors are grateful to Medical Research Center Oulu, University of Oulu Scholarship Foundation for financial support. The Authors would like to thank Riitta Vuento and Erja Tomperi for their expert technical help.

\section{References}

1 Makinen MJ, George SM, Jernvall P, Makela J, Vihko P and Karttunen TJ: Colorectal carcinoma associated with serrated adenoma - prevalence, histological features, and prognosis. J Pathol 193: 286-294, 2001.

2 Young J, Jenkins M, Parry S, Young B, Nancarrow D, English D, Giles G and Jass J: Serrated pathway colorectal cancer in the population: genetic consideration. Gut 56: 1453-1459, 2007.

3 Garcia-Solano J, Perez-Guillermo M, Conesa-Zamora P, AcostaOrtega J, Trujillo-Santos J, Cerezuela-Fuentes P and Makinen MJ: Clinicopathologic study of 85 colorectal serrated adenocarcinomas: further insights into the full recognition of a new subset of colorectal carcinoma. Hum Pathol 41: 1359-1368, 2010.

4 Makinen MJ: Colorectal serrated adenocarcinoma. Histopathology 50: 131-150, 2007.

5 Stefanius K, Ylitalo L, Tuomisto A, Kuivila R, Kantola T, Sirnio $\mathrm{P}$, Karttunen TJ and Makinen MJ: Frequent mutations of KRAS in addition to $B R A F$ in colorectal serrated adenocarcinoma. Histopathology 58: 679-692, 2011.

6 Tuppurainen K, Makinen JM, Junttila O, Liakka A, Kyllonen AP, Tuominen H, Karttunen TJ and Makinen MJ: Morphology and microsatellite instability in sporadic serrated and non-serrated colorectal cancer. J Pathol 207: 285-294, 2005.

7 Leggett B and Whitehall V: Role of the serrated pathway in colorectal cancer pathogenesis. Gastroenterology 138: 20882100, 2010 .

8 Noffsinger AE: Serrated polyps and colorectal cancer: new pathway to malignancy. Annu Rev Pathol 4: 343-364, 2009.

9 Snover DC: Update on the serrated pathway to colorectal carcinoma. Hum Pathol 42: 1-10, 2011.

10 Akimoto N, Fujimori T, Mitomi H, Ichikawa K, Tomita S, Tatsuguchi A, Fujimori S, Naito Z and Sakamoto C: Micropapillary pattern at the invasive front and its association with unresectable colorectal carcinomas. Dis Markers 35: 451-455, 2013. 
11 Hannibal CG, Vang R, Junge J, Frederiksen K, Kurman RJ and Kjaer SK: A nationwide study of ovarian serous borderline tumors in Denmark 1978-2002. Risk of recurrence, and development of ovarian serous carcinoma. Gynecol Oncol 144: 174-180, 2017.

12 Yoshizawa A, Motoi N, Riely GJ, Sima CS, Gerald WL, Kris MG, Park BJ, Rusch VW and Travis WD: Impact of proposed IASLC/ATS/ERS classification of lung adenocarcinoma: prognostic subgroups and implications for further revision of staging based on analysis of 514 stage I cases. Mod Pathol 24: 653-664, 2011.

13 Altieri DC: Survivin, cancer networks and pathway-directed drug discovery. Nat Rev Cancer 8: 61-70, 2008.

14 Altieri DC: Survivin and IAP proteins in cell-death mechanisms. Biochem J 430: 199-205, 2010.

15 Mita AC, Mita MM, Nawrocki ST and Giles FJ: Survivin: key regulator of mitosis and apoptosis and novel target for cancer therapeutics. Clin Cancer Res 14: 5000-5005, 2008.

16 Altieri DC: Survivin, versatile modulation of cell division and apoptosis in cancer. Oncogene 22: 8581-8589, 2003.

17 Gianani R, Jarboe E, Orlicky D, Frost M, Bobak J, Lehner R and Shroyer KR: Expression of survivin in normal, hyperplastic, and neoplastic colonic mucosa. Hum Pathol 32: 119-125, 2001.

18 Stauber RH, Mann W and Knauer SK: Nuclear and cytoplasmic survivin: molecular mechanism, prognostic, and therapeutic potential. Cancer Res 67: 5999-6002, 2007.

19 Fang YJ, Lu ZH, Wang GQ, Pan ZZ, Zhou ZW, Yun JP, Zhang MF and Wan DS: Elevated expressions of MMP7, TROP2, and survivin are associated with survival, disease recurrence, and liver metastasis of colon cancer. Int J Colorectal Dis 24: 875$884,2009$.

20 Knauer SK, Mann W and Stauber RH: Survivin's dual role: an export's view. Cell Cycle 6: 518-521, 2007.

21 Qi G, Tuncel H, Aoki E, Tanaka S, Oka S, Kaneko I, Okamoto M, Tatsuka M, Nakai $S$ and Shimamoto F: Intracellular localization of survivin determines biological behavior in colorectal cancer. Oncol Rep 22: 557-562, 2009.
22 Guadamillas MC, Cerezo A and Del Pozo MA: Overcoming anoikis - pathways to anchorage-independent growth in cancer. J Cell Sci 124: 3189-3197, 2011.

$23 \mathrm{Kim}$ YN, Koo KH, Sung JY, Yun UJ and Kim H: Anoikis resistance: an essential prerequisite for tumor metastasis. Int $\mathbf{J}$ Cell Biol 2012: 306879, 2012.

24 Kyle AH, Baker JH and Minchinton AI: Targeting quiescent tumor cells via oxygen and IGF-I supplementation. Cancer Res 72: 801-809, 2012.

25 Zhang X, de Milito A, Olofsson MH, Gullbo J, D'Arcy P and Linder S: Targeting Mitochondrial Function to Treat Quiescent Tumor Cells in Solid Tumors. Int J Mol Sci 16: 27313-27326, 2015.

26 Masunaga S and Ono K: Significance of the response of quiescent cell populations within solid tumors in cancer therapy. J Radiat Res 43: 11-25, 2002.

27 Hernandez JM, Farma JM, Coppola D, Hakam A, Fulp WJ, Chen DT, Siegel EM, Yeatman TJ and Shibata D: Expression of the antiapoptotic protein survivin in colon cancer. Clin Colorectal Cancer 10: 188-193, 2011.

$28 \mathrm{Li} \mathrm{F}$, Yang J, Ramnath N, Javle MM and Tan D: Nuclear or cytoplasmic expression of survivin: what is the significance? Int J Cancer 114: 509-512, 2005.

29 Negroni A, Cucchiara S and Stronati L: Apoptosis, necrosis, and necroptosis in the gut and intestinal homeostasis. Mediators Inflamm 2015: 250762, 2015.

30 Parfitt JR and Driman DK: Survivin and hedgehog protein expression in serrated colorectal polyps: an immunohistochemical study. Hum Pathol 38: 710-717, 2007.

Received March 7, 2018

Revised March 26, 2018

Accepted March 28, 2018 